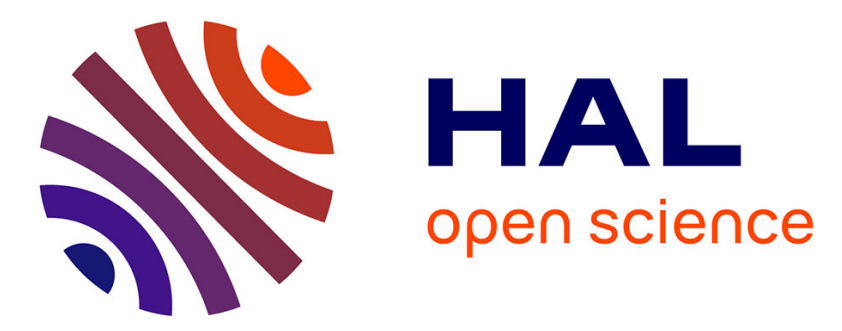

\title{
Study of the simple extension tear test sample for rubber with Configurational Mechanics \\ Erwan Verron
}

\section{To cite this version:}

Erwan Verron. Study of the simple extension tear test sample for rubber with Configurational Mechanics. International Journal of Fracture, 2007, 147 (1 4), pp.219-225. 10.1007/s10704-007-9165-3 . hal-01007346

\section{HAL Id: hal-01007346 \\ https://hal.science/hal-01007346}

Submitted on 16 Jun 2014

HAL is a multi-disciplinary open access archive for the deposit and dissemination of scientific research documents, whether they are published or not. The documents may come from teaching and research institutions in France or abroad, or from public or private research centers.
L'archive ouverte pluridisciplinaire HAL, est destinée au dépôt et à la diffusion de documents scientifiques de niveau recherche, publiés ou non, émanant des établissements d'enseignement et de recherche français ou étrangers, des laboratoires publics ou privés. 


\title{
Study of the simple extension tear test sample for rubber with Configurational Mechanics
}

\author{
Erwan Verron
}

Institut de Recherche en Génie Civil et Mécanique, UMR CNRS 6183, École Centrale de Nantes, BP 92101, 44321 Nantes cedex 3, France

e-mail: erwan.verron@ec-nantes.fr

\begin{abstract}
The simple extension tear test-piece also referred to as the trousers sample is widely used to study crack propagation in rubber. The corresponding energy release rate, called tearing energy for rubber materials, was first established by Rivlin and Thomas ( $J$ Polym Sci, 10:291-318, 1953); a second derivation was proposed later by Eshelby (In G.C. Sih, H. C. van Elst, and D. Broek, editors, Prospects of Fracture Mechanics, 6984, Leyden, 1975). We show here that the derivation of this result can be advantageously revisited through the scope of Configurational Mechanics. Our approach is based on the rigorous definition of the configurations of the body and on the physical significance of the configurational stress tensor. More precisely, it is demonstrated that the change in energy due to crack growth, and then the tearing energy, is directly related to the components of the configurational stress tensor in the body.
\end{abstract}

Keywords Rubber - Tearing energy · Simple extension tear test $\cdot$ Configurational Mechanics

\section{Introduction}

In his seminal paper, Griffith (1920) proposed a criterion to determine the amount of energy involved during crack propagation in brittle materials. Denoting $d U$ $(<0)$ the change of total energy (change of strain energy and work of external forces) and $d A$ the increase in crack surface during crack growth in the body, the energy release rate $G$ is defined by

$G=-\frac{d U}{d A}$,

and the crack growth criterion can be simply written as

$G<G_{c}$,

where $G_{c}$ is a critical value of the energy release rate directly related to the surface free energy of the material. It should be measured in experiments.

More than 30 years later, Rivlin and Thomas (1953) extended the Griffith theory to rubber-like materials considering the following assumptions:

(a) the approach of Griffith is valid for large strain (in fact, no restriction was formulated in the original paper of Griffith),

(b) irreversible changes in energy due to crack growth take place only in the neighbourhood of the crack tip,

(c) the change in energy is independent of the shape and dimensions of the body. 
Then, authors defined the tearing energy $T$, i.e. the counterpart of $G$ for rubberlike materials, by

$$
T=-\left.\frac{\partial w}{\partial A}\right|_{l},
$$

where $w$ is the strain energy, $A$ is the crack surface and the suffix $\cdot l$ denotes differentiation with constant displacement of the boundaries over which forces are applied. Considering thin samples (uniform thickness $h_{0}$ ) and denoting $c$ the length of the crack, the tearing energy reduces to

$T=-\left.\frac{1}{h_{0}} \frac{\partial w}{\partial c}\right|_{l}$

and the corresponding crack growth criterion is simply $T>T_{c}, T_{c}$ being the critical value of the tearing energy that only depends on the material. Nevertheless, due to assumption (b), $T_{c}$ cannot be directly related to the surface free energy of the elastomer. Moreover, the correctness of the above-mentioned assumption (c) was investigated by several authors by making tearing measurement on thin test pieces of different shapes but of the same material, and examining the constancy of $T_{c}$ values obtained (see Thomas (1994) and the references herein).

In their paper published in 1953, Rivlin and Thomas proposed several experimental samples to perform crack propagation experiments in rubber. One of these samples is the simple extension tear test-piece also referred to as the trousers sample. Authors calculated the corresponding expression for the tearing energy and used this sample to measure the critical value of the tearing energy. Later, Eshelby (1975b) considered the same test-piece to illustrate the relevance of pathindependent integrals for the calculation of configurational forces that drive the evolution of singularities. In his paper, Eshelby proposed a second derivation for the tearing energy of the trousers sample. The present paper discusses a third derivation of this result. It is demonstrated that the tearing energy of the simple extension tear test sample can be easily recovered using the general theory of Configurational Mechanics and more precisely the definition of the configurational stress tensor. Previous derivations are first recalled. Then, our proposal is detailed.

\section{Description of the problem}

The sample is a rectangular thin sheet of length $b$ and width $2 a$; the thickness $h_{0}$ is uniform. The test piece

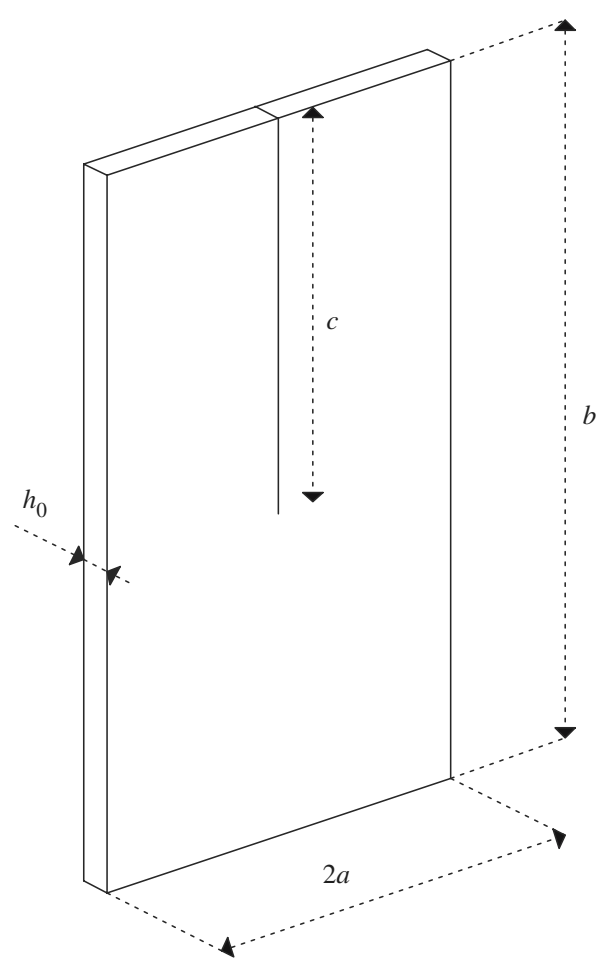

Fig. 1 Trousers sample for rubber tearing

contains a cut of length $c$ (with $c \gg a$ ) parallel to the edges of length $b$. The sample geometry is presented in Fig. 1.

The description of the problem is based on the Fig. 2 . Both the notations proposed by Rivlin and Thomas (1953, p. 302) and the three-dimensional sketch of Greensmith and Thomas (1956, p. 373) (considered later by Eshelby (1975b, p. 76)) are adopted. In the following, the emphasize is laid on the rigorous definition of body configurations. The initial position of the sample shown in Fig. 1 is adopted as the reference configuration $\left(\mathcal{C}_{0}\right)$ (see Fig. 2a). During the experiments, the 'legs of the trousers' are first spread; then, the force $F$ is applied to the legs to produce tearing. It leads to the definition of the deformed configuration $(\mathcal{C})$ as shown in Fig. 2b. In this configuration, the separation between legs extremities is $l$ and the thickness $h$ of the piece is no longer uniform. We consider now that the crack length increases by $d c$ in the deformed configuration, the force $F$ being kept constant. Thus, after unloading, i.e. $F=0$, the sample occupies a new reference configuration $\left(\mathcal{C}_{0}^{\prime}\right)$ depicted in Fig. 2c. The motion between $(\mathcal{C})$ and $\left(\mathcal{C}_{0}^{\prime}\right)$ is defined by its deformation gradient $\mathbf{f}$. 

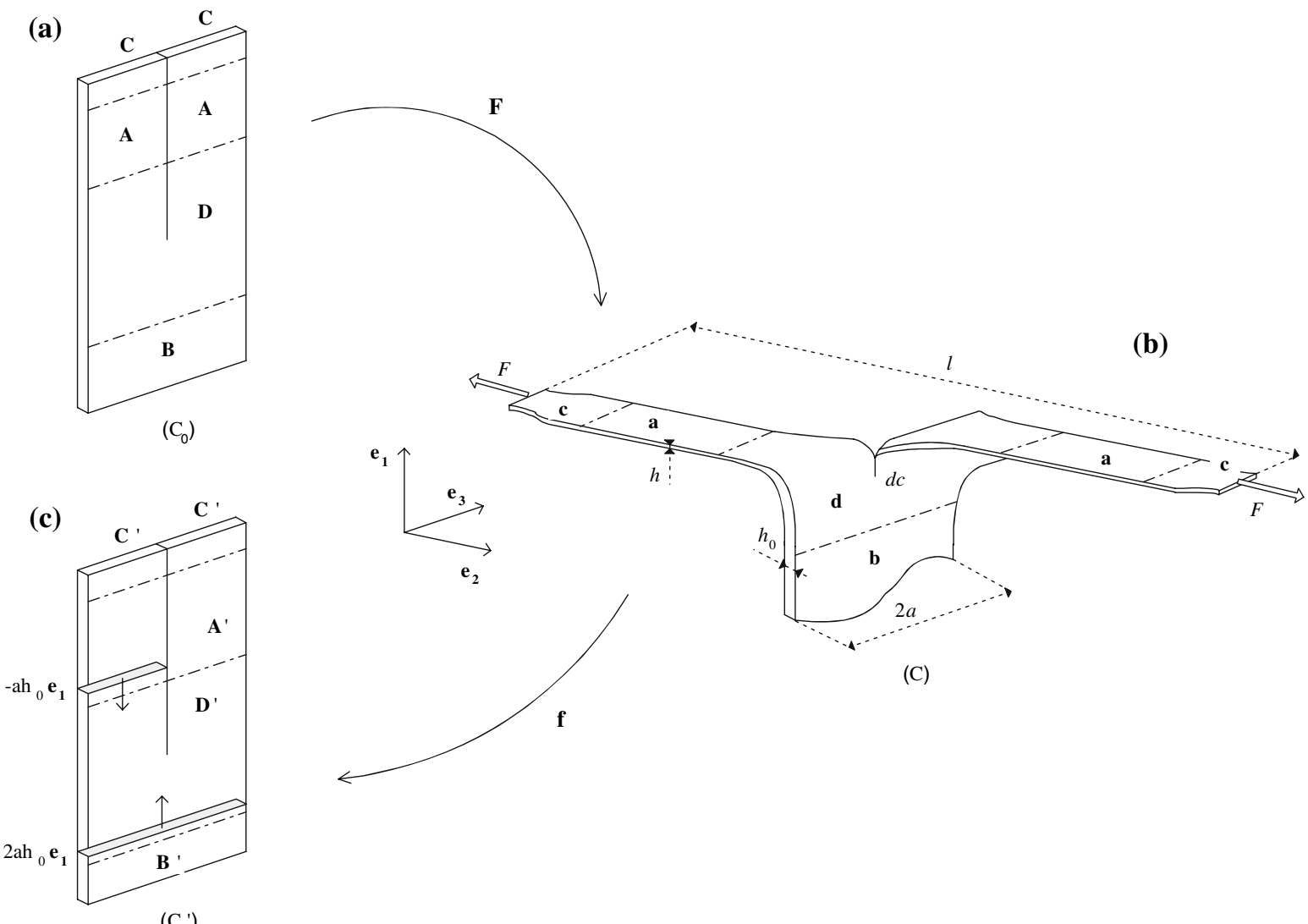

(C)

Fig. 2 Definition of body configurations

Obviously, $\mathbf{f}$ is not exactly equal to $\mathbf{F}^{-1}$ because the crack has grown and $\left(\mathcal{C}_{0}^{\prime}\right)$ differs from $\left(\mathcal{C}_{0}\right)$.

\section{Previous derivations}

\subsection{Derivation of Rivlin and Thomas (1953)}

The determination of the tearing energy as proposed by Rivlin and Thomas (1953) is based on an energetic analysis. For this purpose, authors considered Fig. 2a and $\mathrm{b}$.

The sample can be separated into four different regions in the deformed configuration (Fig. 2b):

- the region a of each of the legs which is substantially in uniaxial extension, the corresponding stretch ratio being denoted $\lambda$,

- the region $\mathbf{b}$ which is substantially undeformed if the uncut part of the sample is sufficiently long,
- the regions $\mathbf{c}$ and the region $\mathbf{d}$ in which strain and stress distributions are complicated, the former correspond to the neighbourhood of the points in which forces apply and the latter corresponds to the neighbourhood of the tip.

When the crack length increases from $c$ to $c+d c$, authors consider that:

- the size of regions a increases and the size of the region $\mathbf{b}$ decreases of the same expense. In the reference configuration (Fig. 2a), the increase in volume of each region $\mathbf{A}$ (which are transformed into regions a by the motion) is $a h_{0} d c$; thus, the decrease in volume of the region $\mathbf{B}$ (which is transformed into region $\mathbf{b}$ by the motion) is equal to $2 a h_{0} d c$,

- the regions $\mathbf{c}$ and the region $\mathbf{d}$ are only moved and their respective sizes (or the sizes of their undeformed counterparts $\mathbf{C}$ and $\mathbf{D}$ ) do not change, 
- moreover, after crack growth the region a is still in uniaxial extension with the same stretch ratio $\lambda$ because $F$ does not change, the region $\mathbf{b}$ is still undeformed, and the strain and stress distributions in regions $\mathbf{c}$ and $\mathbf{d}$ remain unchanged.

So, the length between legs extremities increases by $d l=2 \lambda d c$, and then,

$$
\left.\frac{\partial l}{\partial c}\right|_{F}=2 \lambda \text {. }
$$

Considering that the strain energy of the sample is a function of the two quantities $l$ and $c$, its change $d w$ is related to the changes of crack length $d c$ and overall length $d l$ by

$d w=\left.\frac{\partial w}{\partial l}\right|_{c} d l+\left.\frac{\partial w}{\partial c}\right|_{l} d c$.

Recalling that crack growth is due to the applied force $F$ which remains constant, the previous equation can be written as

$$
\left.\frac{\partial w}{\partial c}\right|_{F}=\left.\left.\frac{\partial w}{\partial l}\right|_{c} \frac{\partial l}{\partial c}\right|_{F}+\left.\frac{\partial w}{\partial c}\right|_{l} .
$$

With respect to the previous statement about the increase in volume of the regions a and the decrease in volume of the region $\mathbf{b}$, the change in energy can be determined in the reference configuration $\left(\mathcal{C}_{0}\right)$,

$$
\left.\frac{\partial w}{\partial c}\right|_{F}=W 2 a h_{0},
$$

where $W$ stands for the strain energy density per unit of undeformed volume and $2 a h_{0}$ is the cross-section of the undeformed sample. Moreover, the force $F$ is related to the change in energy by

$$
\left.\frac{\partial w}{\partial l}\right|_{c}=F \text {. }
$$

Finally, recalling Eqs. 5, 8 and 9, Eq. 7 becomes

$$
\left.\frac{\partial w}{\partial c}\right|_{l}=W 2 a h_{0}-F 2 \lambda,
$$

the tearing energy being obviously deduced from Eq. 4 .

\subsection{Derivation of Eshelby (1975b)}

In order to highlight the connection between the configurational stress tensor (also called the elastic energy momentum tensor or the Eshelby stress tensor) and the path-independent integral for energy release rate,
Eshelby (1975b) proposed a new derivation of the tearing energy for the trousers sample.

In this way, following Knowles and Sternberg (1972) he calculated the following surface integral defined in the reference configuration $\left(\mathcal{C}_{0}\right)$ shown in Fig. 2a

$\gamma_{1}=\int_{S}\left(W-P_{i 1} u_{i, 1}\right) d S$

where $S$ is a surface embracing the tip of the crack, $\mathbf{P}$ is the first Piola-Kirchhoff stress tensor and $\mathbf{u}$ is the displacement vector. In fact, this scalar quantity corresponds to the first component of the configurational force

$\boldsymbol{\gamma}=\int_{S} \overline{\mathbf{\Sigma}} \mathbf{n} d S$

in which $\mathbf{n}$ is the unit vector normal to the surface $d S$ and $\overline{\boldsymbol{\Sigma}}$ is the energy momentum (configurational stress) tensor proposed by Eshelby $(1951,1975 a)$

$\overline{\mathbf{\Sigma}}=W \mathbf{I}-\nabla_{\mathbf{X}}^{t} \mathbf{u} \mathbf{P}$,

where $\mathbf{I}$ is the $3 \times 3$ identity tensor and the superscript ${ }^{t}$ denotes the transposition. $\gamma_{1}$ being path-independent, the author considers a surface $S$ made up of parts of the specimen surface with normal $\mathbf{e}_{2}$ and $\mathbf{e}_{3}$ in regions $\mathbf{A}$, $\mathbf{D}$ and $\mathbf{B}$; a cross-section of the region $\mathbf{B}$ (which is not deformed); and cross-sections of both legs (in regions A). The two first contributions are equal to zero; then $\gamma_{1}$ reduces to

$\gamma_{1}=2\left(W-P_{21} \frac{\partial u_{2}}{\partial X_{1}}\right) a h_{0}$.

A given point with initial coordinates $\left(X_{1}, X_{2}=0\right)$ being first swung round then stretched, the displacement in the $\mathbf{e}_{2}$-direction is

$u_{2}= \pm \lambda X_{1}-X_{2}$,

where the sign depends on the leg: plus sign for the right-hand side leg and minus sign for the left-hand side leg (see Fig. 2). The stress is given by $P_{21}= \pm F / a h_{0}$, with the same remark for the signs. Thus, $\gamma_{1}$ reduces to

$\gamma_{1}=2\left(W a h_{0}-F \lambda\right)$.

Recalling that the total energy release rate (tearing energy) is related to $\gamma_{1}$ by

$T=-\frac{1}{h_{0}} \gamma_{1}$,

in which the minus sign merely indicates that the tear will run to the bottom, the result of Rivlin and Thomas given by Eqs. 4 and 10 is recovered. 


\section{A new derivation}

Our method to determine the tearing energy of the trousers test sample is based on the general theory of Configurational Mechanics. Before presenting our approach, some basic results concerning the configurational stress tensor are recalled. Only results necessary for our derivation are given, for more details the reader can refer to Maugin $(1993,1995)$.

\subsection{Basic definitions}

The configurational stress tensor permits to quantify the evolution of the reference configuration of a given body subject to deformation. This tensor, denoted $\boldsymbol{\Sigma}$ through the rest of the paper, is defined as

$\Sigma=W \mathbf{I}-\mathbf{F}^{t} \mathbf{P}$,

where $\mathbf{F}$ is the deformation gradient (equal to $\mathbf{I}+\nabla_{\mathbf{X}} \mathbf{u}$ ). Note that the expression of the configurational stress tensor is different than the one considered by Eshelby (1975b) and given in Eq. 13, the displacement gradient being replaced by the deformation gradient (see Sect. 4.3 for comments).

The derivation will be essentially based on the physical significance of the components of $\boldsymbol{\Sigma}$. Considering the material space $\mathcal{M}^{3}$ in which the body is defined as a set of particles (a reference configuration), the scalar $d \mathbf{U} \cdot \boldsymbol{\Sigma} d S_{0} \mathbf{N}_{0}$ is the change in energy due to a material displacement, i.e. a displacement in $\mathcal{M}^{3}, d \mathbf{U}$ of the material surface $d S_{0} \mathbf{N}_{0}$. So, as previously established by Kienzler and Herrmann (1997) for small strain, $\Sigma_{i j}$ is the change in energy due to a unit material translation in the direction of the vector $\mathbf{e}_{\mathbf{i}}$ of a unit material surface which normal vector is $\mathbf{e}_{\mathbf{j}}$.

\subsection{Application to the trousers test sample}

As recalled above, the tearing energy is related to the change in energy between a body with a crack of length $c$ and the same body with a crack of length $c+d c$ for a given motion. In order to determine the tearing energy for the trousers sample, let us examine the Fig. 2. In this figure, the configurations $\left(\mathcal{C}_{0}\right)$ (Fig. 2a) and $\left(\mathcal{C}_{0}^{\prime}\right)$ (Fig. 2c) are two reference configurations; then, with the help of the configurational stress tensor, it is possible to calculate the change in energy between them. In this way, only the material transformation, i.e. defined in $\mathcal{M}^{3}$, between $\left(\mathcal{C}_{0}\right)$ and $\left(\mathcal{C}_{0}^{\prime}\right)$ has to be considered, the forces applied to the sample being known (Fig. 2b).

The analysis of the problem proposed by Rivlin and Thomas (1953) and recalled in Sect. 3.1 is adopted; regions $\mathbf{A}, \mathbf{B}, \mathbf{C}$ and $\mathbf{D}$ of $\left(\mathcal{C}_{0}\right)$ are transformed into $\mathbf{A}^{\prime}, \mathbf{B}^{\prime}, \mathbf{C}^{\prime}$ and $\mathbf{D}^{\prime}$ of $\left(\mathcal{C}_{0}^{\prime}\right)$ in the following manner:

- the two regions $\mathbf{A}$ are transformed into the two regions $\mathbf{A}^{\prime}$ by the material translation $-d c \mathbf{e}_{\mathbf{1}}$ of the material surface $a h_{0}\left(-\mathbf{e}_{1}\right)$ (grey cross-section in Fig. 2c),

- the region $\mathbf{B}$ is transformed into the region $\mathbf{B}^{\prime}$ by the material translation $-d c \mathbf{e}_{\mathbf{1}}$ of the material surface $2 a h_{0} \mathbf{e}_{\mathbf{1}}$ (grey cross-section in Fig. 2c),

- the regions $\mathbf{C}$ and $\mathbf{D}$ are transformed into regions $\mathbf{C}^{\prime}$ et $\mathbf{D}^{\prime}$ by a 'rigid body material motion'.

Then, the change in energy between configurations $\left(\mathcal{C}_{0}\right)$ and $\left(\mathcal{C}_{0}^{\prime}\right)$ is

$d w=d w_{\mathbf{A} \rightarrow \mathbf{A}^{\prime}}+d w_{\mathbf{B} \rightarrow \mathbf{B}^{\prime}}+d w_{\mathbf{C} \rightarrow \mathbf{C}^{\prime}}+d w_{\mathbf{D} \rightarrow \mathbf{D}^{\prime}}$

with

$$
\begin{aligned}
d w_{\mathbf{A} \rightarrow \mathbf{A}^{\prime}} & =2\left[-d c \mathbf{e}_{\mathbf{1}} \cdot \boldsymbol{\Sigma}^{\mathbf{A}} a h_{0}\left(-\mathbf{e}_{\mathbf{1}}\right)\right] \\
& =2 d c a h_{0} \Sigma_{11}^{\mathbf{A}}, \\
d w_{\mathbf{B} \rightarrow \mathbf{B}^{\prime}} & =-d c \mathbf{e}_{\mathbf{1}} \cdot \boldsymbol{\Sigma}^{\mathbf{B}} 2 a h_{0} \mathbf{e}_{\mathbf{1}} \\
& =-2 d c a h_{0} \Sigma_{11}^{\mathbf{B}},
\end{aligned}
$$

and

$d w_{\mathbf{C} \rightarrow \mathbf{C}^{\prime}}=0$ and $d w_{\mathbf{D} \rightarrow \mathbf{D}^{\prime}}=0$.

In these equations $\boldsymbol{\Sigma}^{\mathbf{A}}$ and $\boldsymbol{\Sigma}^{\mathbf{B}}$ stand for the values of the configurational stress tensor in regions $\mathbf{A}$ and $\mathbf{B}$, respectively. Equation 22 is obvious because regions $\mathbf{C}$ and $\mathbf{D}$ are statically moved in $\mathcal{M}^{3}$ and consequently their energies are unchanged. Moreover, the region $\mathbf{B}$ being undeformed and stress-free, $\boldsymbol{\Sigma}^{\mathbf{B}}=\mathbf{0}$, and

$d w_{\mathbf{B} \rightarrow \mathbf{B}^{\prime}}=0$.

Finally, the change in energy of the body is only due to the transformation of the regions $\mathbf{A}$ into the regions $\mathbf{A}^{\prime}$. These regions being in simple extension and assuming that the material is incompressible, the deformation gradient and the first Piola-Kirchhoff stress tensor are: 
- for the right-hand side leg of the trousers

$$
\mathbf{F}=\lambda \mathbf{e}_{\mathbf{2}} \otimes \mathbf{e}_{\mathbf{1}}-\lambda^{-\frac{1}{2}} \mathbf{e}_{\mathbf{1}} \otimes \mathbf{e}_{\mathbf{2}}+\lambda^{-\frac{1}{2}} \mathbf{e}_{\mathbf{3}} \otimes \mathbf{e}_{\mathbf{3}}
$$

and

$$
\mathbf{P}=\frac{F}{a h_{0}} \mathbf{e}_{\mathbf{2}} \otimes \mathbf{e}_{\mathbf{1}},
$$

- for the left-hand side leg of the trousers

$$
\mathbf{F}=-\lambda \mathbf{e}_{\mathbf{2}} \otimes \mathbf{e}_{\mathbf{1}}+\lambda^{-\frac{1}{2}} \mathbf{e}_{\mathbf{1}} \otimes \mathbf{e}_{\mathbf{2}}+\lambda^{-\frac{1}{2}} \mathbf{e}_{\mathbf{3}} \otimes \mathbf{e}_{\mathbf{3}}(26)
$$

and

$$
\mathbf{P}=-\frac{F}{a h_{0}} \mathbf{e}_{\mathbf{2}} \otimes \mathbf{e}_{\mathbf{1}} .
$$

For both legs, the first component of $\boldsymbol{\Sigma}^{\mathbf{A}}$ reduces to

$$
\Sigma_{11}^{\mathbf{A}}=W-F_{j 1} P_{j 1}=W-\lambda \frac{F}{a h_{0}} .
$$

So, the change in energy of the regions $\mathbf{A}$ is

$$
\begin{aligned}
d w_{\mathbf{A} \rightarrow \mathbf{A}^{\prime}} & =2 d c\left(W-\lambda \frac{F}{a h_{0}}\right) a h_{0} \\
& =\left(W 2 a h_{0}-F 2 \lambda\right) d c .
\end{aligned}
$$

So considering Eqs. 19, 22 and 23, it is also equal to the total change in energy between configurations $\left(\mathcal{C}_{0}\right)$ and $\left(\mathcal{C}_{0}^{\prime}\right)$. It can be written as

$$
\frac{\partial w}{\partial c}=W 2 a h_{0}-F 2 \lambda \text {. }
$$

So the result due to Rivlin and Thomas, and Eshelby is recovered.

\subsection{Remark on the definition of the configurational stress tensor}

It should be noted that the derivation proposed above does not depend on the choice of the reference configuration. As an example, consider the configuration depicted in Fig. 3. During experiments, the legs of the trousers are first spread then extended. Thus this configuration is undeformed and stress-free because the motion between the configuration $\left(\mathcal{C}_{0}\right)$ shown in Fig. 2a and this one reduces to a rigid body motion of a part of the sample. So, it can be adopted as a reference configuration. In this case, the change in energy between regions $\mathbf{A}$ and $\mathbf{A}^{\prime}$ is simply due to a material unit translation $-d c \mathbf{e}_{2}$ of the material surface $a h_{0}\left(-\mathbf{e}_{2}\right)$ for the right-hand side leg and to a material unit translation

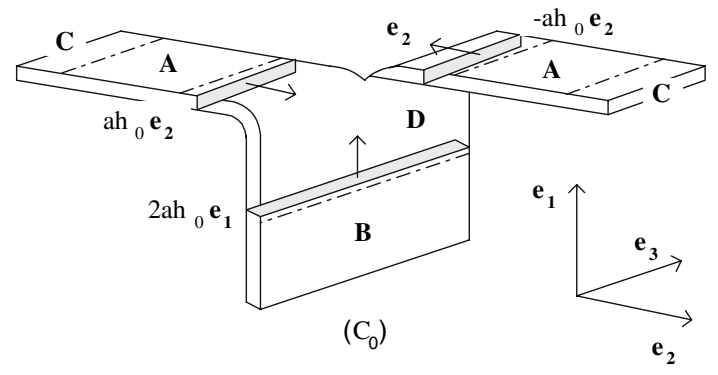

Fig. 3 A new reference configuration for the trousers sample

$d c \mathbf{e}_{2}$ of the material surface $a h_{0} \mathbf{e}_{2}$ for the right-hand side leg (see the grey cross-sections in Fig. 3)

$$
\begin{aligned}
d w_{\mathbf{A} \rightarrow \mathbf{A}^{\prime}}= & d c \mathbf{e}_{\mathbf{2}} \cdot \boldsymbol{\Sigma}^{\mathbf{A}} a h_{0} \mathbf{e}_{\mathbf{2}} \\
& +d c\left(-\mathbf{e}_{2}\right) \cdot \boldsymbol{\Sigma}^{\mathbf{A}} a h_{0}\left(-\mathbf{e}_{2}\right) \\
= & 2 d c a h_{0} \Sigma_{22}^{\mathbf{A} .}
\end{aligned}
$$

The changes in energy due to the other regions remain equal to zero. For both legs of the sample, the deformation gradient and the first Piola-Kirchhoff stress tensor are identical and respectively given by

$\mathbf{F}=\lambda \mathbf{e}_{\mathbf{2}} \otimes \mathbf{e}_{\mathbf{2}}+\lambda^{-\frac{1}{2}} \mathbf{e}_{\mathbf{1}} \otimes \mathbf{e}_{\mathbf{1}}+\lambda^{-\frac{1}{2}} \mathbf{e}_{\mathbf{3}} \otimes \mathbf{e}_{\mathbf{3}}$

and

$\mathbf{P}=\frac{F}{a h_{0}} \mathbf{e}_{\mathbf{2}} \otimes \mathbf{e}_{2}$.

With these expressions, the result Eq. 30 is easily recovered.

As noted above, Eshelby (1975b) considered the configurational stress tensor $\bar{\Sigma}$ defined in terms of the displacement gradient (Eq. 13) rather than $\boldsymbol{\Sigma}$ defined in terms of the deformation gradient (Eq. 18). It is to note that Knowles and Sternberg (1972) defined several $J$-type invariant integrals for elastostatic finite deformations and especially the integrals based on both configurational stress tensors. The former definition of the configurational stress tensor (Eq. 13) was first proposed by Eshelby (1951) for small strain but its use for large strain problems is revealed inappropriate (Maugin, 1993). In fact, this can be illustrated by the present example. Indeed, adopting the tensor $\overline{\boldsymbol{\Sigma}}$ and making the derivation with the reference configuration shown in Fig. 2a leads to the right expression of the tearing energy. Nevertheless, making it with the reference configuration of Fig. 3 fails. 


\section{Conclusion}

In this paper, the derivation of the energy release rate of the simple extension tear sample was revisited in the framework of Configurational Mechanics. Obviously, such a derivation is possible because of the simplicity of the mechanical fields in the different regions of the sample: the tearing energy reduces to the value of one of the component of the tensor and does not involve a surface integral. In more general cases, the use of path-integrals, i.e. $J$-type integrals, is mandatory because the configurational stress tensor, i.e. the integrant, is not uniform. Nevertheless, such a study is useful to illustrate the relevance of the configurational mechanics quantities to determine changes in energy between different reference configurations of a given body. Moreover, this type of approach can be considered to design new test samples for fracture mechanics: similarly to the simple tests for the mechanical response of materials (uniaxial extension, simple shear, equibiaxial extension ...), new tear samples can be proposed by studying the configurational stress field and the possibility to calculate it analytically. Moreover, it has been shown here that the components of $\boldsymbol{\Sigma}$ are appropriate quantities for the study of macroscopic cracks in rubberlike materials. Motivated by this small study, the tensor can be considered as an efficient tool for the study of the evolution of microscopic defects in elastomers; such an approach was recently proposed by Verron et al., 2006, Verron and Andriyana, In Press, Andriyana and Verron, 2007) to predict fatigue crack initiation in rubber under multiaxial loading conditions.

\section{References}

Andriyana A, Verron E (2007) Prediction of fatigue life improvement in natural rubber using configurational stress. Int $\mathbf{J}$ Solids Struct 44:2079-2092

Eshelby JD (1951) The force on an elastic singularity. Phil Trans R Soc Lond A 244:87-112

Eshelby JD (1975a) The elastic energy-momentum tensor. J Elast 5(3-4):321-335

Eshelby JD (1975b) The calculation of energy release rates. In: Sih GC, van Elst HC, Broek D (eds) Prospects of fracture mechanics. Noordhoff, Leyden pp 69-84

Greensmith HW, Thomas AG (1956) Rupture of rubber - III Determination of tear properties. Rubber Chem Technol 29:372-381

Griffith AA (1920) The phenomena of rupture and flow in solids. Phil Trans R Soc Lond A 221:163-198

Kienzler R, Herrmann G (1997) On the properties of the Eshelby tensor. Acta Mech 125:73-91

Knowles JK, Sternberg E (1972) On a class of conservation laws in linearized and finite elastostatics. Arch Rat Mech Anal 44:187-211

Maugin GA (1993) Material inhomogeneities in elasticity. Chapman and Hall, London

Maugin GA (1995) Material forces: concepts and applications. Appl Mech Rev 48:213-245

Rivlin RS, Thomas AG (1953) Rupture of rubber. I. Characteristic energy for tearing. J Polym Sci 10:291-318

Thomas AG (1994) The development of fracture mechanics for elastomers. Rubber Chem Technol 67:G50-G60

Verron E, Andriyana A (In Press) Definition of a new predictor for multiaxial fatigue crack nucleation in rubber. J Mech Phys Solids. doi:10.1016/j.jmps.2007.05.019

Verron E, Le Cam J-B, Gornet L (2006) A multiaxial criterion for crack nucleation in rubber. Mech Res Commun 33:493-498 